\title{
Consensus of Multiagent Systems with Directed Topology and Communication Time Delay Bases on the Laplace Transform
}

\author{
Bo Liu, ${ }^{1}$ Li Wang, ${ }^{2}$ Dehui Sun, ${ }^{2}$ and Xinmao $\mathrm{Zhu}^{1}$ \\ ${ }^{1}$ College of Science, North China University of Technology, Beijing 100144, China \\ ${ }^{2}$ Key Laboratory of Beijing for Field-Bus Technology \& Automation, North China University of Technology, Beijing 100144, China \\ Correspondence should be addressed to Bo Liu; liuboangle@163.com
}

Received 6 May 2014; Accepted 18 June 2014; Published 1 July 2014

Academic Editor: Wei Zhang

Copyright (c) 2014 Bo Liu et al. This is an open access article distributed under the Creative Commons Attribution License, which permits unrestricted use, distribution, and reproduction in any medium, provided the original work is properly cited.

\begin{abstract}
This paper investigates the consensus problem of multiagent systems with directed topologies. Different from the literatures, a new method, the Laplace transform, to study the consensus of multiagent systems with directed topology and communication time delay is proposed. The accurate state of the consensus center and the upper bound of the communication delay to make the agents reach consensus are given. It is proved that all the agents could aggregate and eventually form a cohesive cluster in finite time under certain conditions, and the consensus center is only determined by the initial states and the communication configuration among the agents. Finally, simulations are given to illustrate the theoretical results.
\end{abstract}

\section{Introduction}

In recent years, there has been an increasing interest in the study of consensus and rendezvous problems in the multiagent systems [1-16]. This is partly due to the wide applications in cooperative control of unmanned air vehicles, formation control of mobile robots, design of sensor networks, flocking of ants and birds, distributed decision making, and so on [5-10]. Vicsek et al. [1] proposed a discrete model of autonomous agents. Each agent of such model was moving at a constant identical velocity, and the direction was updated via a local rule based on the average of the directions of its neighbors. At the same time, they gave some numerical simulations to describe the dynamic behavior of the model. Jadbabaie et al. [3] gave a theoretical explanation for the numerical results of Vicsek's model. In [5], Olfati-Saber and Murray analyzed the consensus problem of multiagent systems with time delay and obtained the accurate bound of the time delay with undirected topology. In $[7,9]$, the authors discussed the consensus of second-order multiagent systems with time delay by Lyapunov approach. However, due to certain limitations, the bound of delay in those papers is not specific.

The analysis of the coupling topology plays an important part in discussing the consensus problems. Jadbabaie et al. [3] discussed the communication information by applying an undirected graph to model the coupling topology among the agents. Olfati-Saber and Murray [5] investigated the average consensus problem with directed topology, where the coupling topology is undirected and needs to satisfy the balance condition, which is a strong condition for the consensus. Ren and Beard [11] introduced the definition of spanning tree to depict the coupling topology. It was shown that consensus can be achieved asymptotically if the directed interaction graph contains a spanning tree as the system evolves. A useful Lemma about the Laplacian matrix was given in [11], and Laplacian matrix has a simple zero eigenvalue if and only if the directed graph has a spanning tree. Lin et al. [12] introduced the definition of globally reachable node to describe the coupling topology and gave a similar lemma about the Laplacian matrix; that is, the digraph has a globally reachable node if and only if 0 is a simple eigenvalue of Laplacian matrix.

In this paper, we discuss the consensus problem of the multiagent system mentioned in [5] with directed topology and time delay. Compared with the previous references, the main contribution of this paper is to study the coupling topology in a more general case. According to the Laplace transform, we can not only have the specific consensus center of the model but also obtain the accurate upper bound of 
the communication delay value to achieve consensus. By the Laplace transform, we can eliminate some assumptions used in the Lyapunov approach and give further simplification of the assumptions.

This paper is organized as follows. Section 2 presents some preliminaries of graph theory. Section 3 proposes the model and gives the analysis for the consensus of the model. In Section 3, some extensions of the studies are discussed. Section 4 gives simulations to verify the theoretical results. Finally, we summarize our main contribution in Section 5.

\section{Some Preliminaries}

To discuss the coupling topology of the communication configuration of the agents, graph theory is a very effective tool. If each agent is regarded as a node, then the coupling topology is conveniently described by a directed graph. In particular, in the definition of the directed graph, self-loops are excluded. Let $\mathscr{G}=(\mathscr{V}, \mathscr{E}, A)$ be a weighted digraph of order $n$ with the set of nodes $\mathscr{V}=\{1,2,3, \ldots, n\}$ and set of arcs $\mathscr{E} \subseteq \mathscr{V} \times \mathscr{V}$, and $A=\left[a_{i j}\right]$ is the adjacency matrix of graph $\mathscr{G}$. An $\operatorname{arc}$ of $\mathscr{G}$ is denoted by $(i, j)$, which is from $i$ to $j$. The set of neighbors of node $i$ is denoted by $\mathcal{N}_{i}=\{j \in \mathscr{V}$ : $(i, j) \in \mathscr{E}\}$. A digraph $\mathscr{G}$ is strongly connected if there exists a path between any two distinct nodes. For a node $j$, if there exists at least a path from every other node $i$ in $\mathscr{G}$ to node $j$, we say that node $j$ is globally reachable.

A diagonal matrix $D=\operatorname{diag}\left\{d_{1}, d_{2}, \ldots, d_{n}\right\} \in R^{n \times n}$ is a degree matrix of $\mathscr{G}$, and its diagonal elements $d_{i}=\sum_{j \in \mathcal{N}_{i}} a_{i j}$ for $i=1,2, \ldots, n$. The Laplacian of the weighted digraph $\mathscr{G}$ (or matrix $A$ ) is denoted as

$$
L=D-A \in R^{n \times n}
$$

It is obvious that the digraph $\mathscr{G}$, the adjacency matrix $A$, and the Laplacian matrix $L$ are peer-to-peer. Some basic properties of the Laplacian matrix need to be introduced in the following.

Lemma 1 (see [12]). The digraph $\mathscr{G}$ has a globally reachable node if and only if the Laplacian matrix $L$ has a simple zero eigenvalue (with eigenvector $\mathbf{1}=(1,1, \ldots, 1)^{T} \in \mathbb{R}^{n}$ ).

Lemma 2 (see [5]). The nonzero eigenvalues of L are of positive real part.

Lemma 3. For arbitrary row of the Laplacian matrix $L$, the cofactor of any elements is equal.

Proof. For Laplacian $L$

$$
\left(\begin{array}{cccc}
l_{11} & l_{12} & \cdots & l_{1 n} \\
l_{21} & l_{22} & \cdots & l_{2 n} \\
\vdots & \vdots & \ddots & \vdots \\
l_{n 1} & l_{n 2} & \cdots & l_{n n}
\end{array}\right)
$$

without loss of generality, the first row of $L$ is chosen to discuss. The cofactors of element $l_{1 j}$ and $l_{1(j+1)}, j \in$ $\{1,2, \ldots, n-1\}$, are, respectively, denoted by

$$
\begin{aligned}
& M_{1 j}=(-1)^{1+j}\left|\begin{array}{ccccccc}
l_{21} & l_{22} & \cdots & l_{2(j-1)} & l_{2(j+1)} & \cdots & l_{2 n} \\
l_{31} & l_{32} & \cdots & l_{3(j-1)} & l_{3(j+1)} & \cdots & l_{3 n} \\
\vdots & \vdots & \cdots & \vdots & \vdots & \ddots & \vdots \\
l_{n 1} & l_{n 2} & \cdots & l_{n(j-1)} & l_{n(j+1)} & \cdots & l_{n n}
\end{array}\right|, \\
& M_{1(j+1)}=(-1)^{1+j+1}\left|\begin{array}{ccccccc}
l_{21} & l_{22} & \cdots & l_{2 j} & l_{2(j+2)} & \cdots & l_{2 n} \\
l_{31} & l_{32} & \cdots & l_{3 j} & l_{3(j+2)} & \cdots & l_{3 n} \\
\vdots & \vdots & \cdots & \vdots & \vdots & \ddots & \vdots \\
l_{n 1} & l_{n 2} & \cdots & l_{n j} & l_{n(j+2)} & \cdots & l_{n n}
\end{array}\right| \text {. }
\end{aligned}
$$

For $M_{1(j+1)}$, adding all the other columns to the $j$ th column, we can have

$$
\begin{aligned}
& M_{1(j+1)} \\
& =(-1)^{1+j+1}\left|\begin{array}{ccccccc}
l_{21} & l_{22} & \cdots & -l_{2(j+1)} & l_{2(j+2)} & \cdots & l_{2 n} \\
l_{31} & l_{32} & \cdots & -l_{3(j+1)} & l_{3(j+2)} & \cdots & l_{3 n} \\
\vdots & \vdots & \cdots & \vdots & \vdots & \ddots & \vdots \\
l_{n 1} & l_{n 2} & \cdots & -l_{n(j+1)} & l_{n(j+2)} & \cdots & l_{n n}
\end{array}\right| \\
& =(-1)^{1+j+1+1}\left|\begin{array}{ccccccc}
l_{21} & l_{22} & \cdots & l_{2(j+1)} & l_{2(j+2)} & \cdots & l_{2 n} \\
l_{31} & l_{32} & \cdots & l_{3(j+1)} & l_{3(j+2)} & \cdots & l_{3 n} \\
\vdots & \vdots & \cdots & \vdots & \vdots & \ddots & \vdots \\
l_{n 1} & l_{n 2} & \cdots & l_{n(j+1)} & l_{n(j+2)} & \cdots & l_{n n}
\end{array}\right| \\
& =M_{1 j} \text {. }
\end{aligned}
$$

Since $j$ is arbitrary, we can obtain $M_{11}=M_{12}=\cdots=M_{1 n}$. Similarly, we can have $M_{21}=M_{22}=\cdots=M_{2 n}, \cdots, M_{n 1}=$ $M_{n 2}=\cdots=M_{n n}$.

This completes the proof.

\section{Model Formulation and Analysis}

Consider a multiagent system consisting of $n$ agents in $m$ dimensional Euclidian space whose motion is governed by the following delay differential equations:

$$
\dot{x}_{i}(t)=-\sum_{j \in \mathcal{N}_{i}} a_{i j}\left(x_{i}(t-\tau)-x_{j}(t-\tau)\right), \quad i=1,2, \ldots, n,
$$

where $x_{i} \in \mathbb{R}^{m}$ represents the state of agent $i, \mathcal{N}_{i}$ is the neighbor set of agent $i, A=\left(a_{i j}\right)$ is the coupling weight matrix, and $a_{i j}$ is weight parameter with $a_{i j}>0$, if agent $i$ has information with agent $j$; otherwise, $a_{i i}=0$; the time delay $\tau>0$ is a constant.

For simplicity of discussion, we take $m=1$ in model (5). Then, model (5) can be rewritten into a matrix form:

$$
\dot{X}(t)=-L X(t-\tau),
$$

where $X(t)=\left(x_{1}(t), x_{2}(t), \ldots, x_{n}(t)\right)^{T} \in R^{n}$ and $L$ is the Laplacian matrix. 
For system (6), we can have the following main results by Laplace transform.

Theorem 4. For system (6), if there exists at least one globally reachable node in $\mathscr{G}$ and the time delay parameter $\tau$ satisfies

$$
\tau<\min _{i=2,3, \ldots, n} \frac{\pi / 2-\left|\arg \left(\lambda_{i}\right)\right|}{\left\|\lambda_{i}\right\|},
$$

then the states of all agents of the system will asymptotically converge to a constant value; that is,

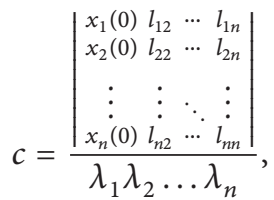

where $\lambda_{1} \lambda_{2} \ldots \lambda_{n}$ are the nonzero eigenvalues of $L$ and $\arg \left(\lambda_{i}\right)$ and $\left\|\lambda_{i}\right\|$ are the argument and modulus of $\lambda_{i}$, respectively.

Proof. Applying Laplace transform on (6), succinctly denoted as $\mathscr{L}(\cdot)$, we can obtain

$$
\begin{aligned}
s \mathscr{L}(X(t))-X(0) & =-L \int_{0}^{\infty} e^{-s t} X(t-\tau) d t \\
& =-L \int_{\tau}^{\infty} e^{-s t} X(t-\tau) d t \\
& =-L \int_{0}^{\infty} e^{-s(\xi+\tau)} X(t) d \xi \\
& =-e^{-s \tau} L \mathscr{L}(X(t)) ;
\end{aligned}
$$

that is,

$$
\left(s I+e^{-s \tau} L\right) \mathscr{L}(X(t))=X(0),
$$

where $s$ is the Laplace variable. For simplicity, $\mathscr{L} X(t)$ is denoted as $\mathscr{X}(s)$.

According to Cramer rule, we can get the solutions of (10):

$$
\mathscr{X}_{i}(s)=\frac{\Delta\left(s I+e^{-s \tau} L\right)^{i}}{\Delta\left(s I+e^{-s \tau} L\right)}, \quad i=1,2, \ldots, n,
$$

where $\Delta\left(s I+e^{-s \tau} L\right)^{i}$ is the determinant of $s I+e^{-s \tau} L$ in which the $i$ th column has been replaced by $X(0) ; \Delta\left(s I+e^{-s \tau} L\right)$ is the determinant of matrix $\left(s I+e^{-s \tau} L\right)$.

By calculating, we can obtain

$$
\mathscr{X}_{i}(s)=\frac{e^{-(n-1) s \tau} \Delta\left(s e^{s \tau} I+L\right)^{i}}{e^{-n s \tau} \Delta\left(s e^{s \tau} I+L\right)}=\frac{e^{s \tau} \Delta\left(s e^{s \tau} I+L\right)^{i}}{\Delta\left(s e^{s \tau} I+L\right)} .
$$

That is fractional expression about variable $s e^{s \tau}$. If there are common factors between $\Delta\left(s e^{s \tau} I+L\right)^{i}$ and $\Delta\left(s e^{s \tau} I+L\right)$, we can reduce them and make them irreducible. In the following, assume that $\Delta\left(s e^{s \tau} I+L\right)^{i}$ and $\Delta\left(s e^{s \tau} I+L\right)$ are irreducible. For simplicity, we denote $P_{i}(s)=\Delta\left(s e^{s \tau} I+L\right)^{i}$ and $Q(s)=$ $\Delta\left(s e^{s \tau} I+L\right)$.
According to Lemma 1, the zero eigenvalue of $L$ is simple and the eigenvalues of $L$ are denoted as

$$
0, \lambda_{2}, \lambda_{3}, \ldots, \lambda_{h}, \lambda_{j}, \ldots, \lambda_{l}
$$

where $\lambda_{j}, \ldots, \lambda_{l}$ are the multiple eigenvalues corresponding with the multiplicity as $q, \ldots, p$, respectively. Then, it follows that

$$
\begin{aligned}
& \Delta\left(s e^{s \tau} I+L\right) \\
& \quad=s e^{s \tau}\left(s e^{s \tau}+\lambda_{2}\right) \cdots\left(s e^{s \tau}+\lambda_{h}\right)\left(s e^{s \tau}+\lambda_{j}\right)^{p} \cdots\left(s+\lambda_{l}\right)^{q} .
\end{aligned}
$$

By Heaviside's method, (12) can be expanded into

$$
\begin{aligned}
& \mathscr{X}_{i}(s)=e^{s \tau}\left(\frac{c_{1}^{i}}{s e^{s \tau}}+\frac{c_{2}^{i}}{s e^{s \tau}+\lambda_{2}}+\cdots+\frac{c_{h}^{i}}{s e^{s \tau}+\lambda_{h}}\right. \\
& +\frac{c_{j p}^{i}}{\left(s e^{s \tau}+\lambda_{j}\right)^{p}}+\frac{c_{j(p-1)}^{i}}{\left(s e^{s \tau}+\lambda_{j}\right)^{(p-1)}} \\
& +\cdots+\frac{c_{j 1}^{i}}{\left(s e^{s \tau}+\lambda_{j}\right)}+\cdots \\
& +\frac{c_{l q}^{i}}{\left(s e^{s \tau}+\lambda_{l}\right)^{q}}+\frac{c_{l(q-1)}^{i}}{\left(s e^{s \tau}+\lambda_{l}\right)^{(q-1)}} \\
& \left.+\cdots+\frac{c_{l 1}^{i}}{\left(s e^{s \tau}+\lambda_{l}\right)}\right) \\
& \triangleq \frac{c_{1}^{i}}{s}+\frac{c_{2}^{i}}{s+\lambda_{2} e^{-s \tau}}+\cdots+\frac{c_{h}^{i}}{s+\lambda_{h} e^{-s \tau}} \\
& +\frac{c_{j p}^{i}}{\left(s+\lambda_{j} e^{-s \tau}\right)^{p}}+\frac{c_{j(p-1)}^{i}}{\left(s+\lambda_{j} e^{-s \tau}\right)^{(p-1)}} \\
& +\cdots+\frac{c_{j 1}^{i}}{\left(s+\lambda_{j} e^{-s \tau}\right)}+\cdots \\
& +\frac{c_{l q}^{i}}{\left(s+\lambda_{l} e^{-s \tau}\right)^{q}}+\frac{c_{l(q-1)}^{i}}{\left(s+\lambda_{l} e^{-s \tau}\right)^{(q-1)}} \\
& +\cdots+\frac{c_{l 1}^{i}}{\left(s+\lambda_{l} e^{-s \tau}\right)},
\end{aligned}
$$


where

$$
\begin{gathered}
c_{1}^{i}=\left.s \frac{P_{i}(s)}{Q(s)}\right|_{s=0}, \quad c_{e}^{i}=\left.\left(s+\lambda_{e}\right) \frac{P_{i}(s)}{Q(s)}\right|_{s=\lambda_{e}}, \quad e=2, \ldots, h, \\
c_{j f}^{i}=\left.\frac{d^{p-f}}{d s^{p-f}}\left[\left(s+\lambda_{j}\right)^{(p-f)} \frac{P_{i}(s)}{Q(s)}\right]\right|_{s=\lambda_{j}}, \quad f=1,2, \ldots, p, \\
\vdots \\
c_{l g}^{i}=\left.\frac{d^{q-g}}{d s^{q-g}}\left[\left(s+\lambda_{l}\right)^{(q-g)} \frac{P_{i}(s)}{Q(s)}\right]\right|_{s=\lambda_{l}}, \quad g=1,2, \ldots, q .
\end{gathered}
$$

Equation (15) is the transfer function of $x_{i}(t)$; the Laplace reverse transform of the first term is a constant value $c_{1}^{i}$. In order to make $x_{i}(t)$ asymptotically stable, the real part of the denominators' roots must be negative, which implies that the roots of transcendental equation $s+\lambda_{k} e^{-s \tau}=0$ must have negative real part. Then, the roots of

$$
s+\lambda_{k} e^{-s \tau}=0
$$

will be analyzed in the following. To solve (17), we let $s=x+$ $i y$, and $\lambda_{k}=u+i v$, where $u>0$, and $i=\sqrt{-1}$ is the plural unit. Then,

$$
x+i y+u e^{-(x+i y) \tau}+i v e^{-(x+i y) \tau}=0
$$

which can be expanded as

$$
\begin{gathered}
x+i y+u e^{-x \tau}(\cos (-y \tau)+i \sin (-y \tau)) \\
+i v e^{-x \tau}(\cos (-y \tau)+i \sin (-y \tau))=0
\end{gathered}
$$

that is,

$$
\begin{aligned}
x+ & u e^{-x \tau} \cos (-y \tau)-v e^{-x \tau} \sin (-y \tau) \\
& +i\left(y+u e^{-x \tau} \sin (-y \tau)+v e^{-x \tau} \cos (-y \tau)\right)=0 .
\end{aligned}
$$

Separating the real and imaginary parts, then we can have

$$
\begin{aligned}
& x+u e^{-x \tau} \cos (-y \tau)-v e^{-x \tau} \sin (-y \tau)=0, \\
& y+u e^{-x \tau} \sin (-y \tau)+v e^{-x \tau} \cos (-y \tau)=0 .
\end{aligned}
$$

Solving the above equations, we can get

$$
\begin{aligned}
& x+\sqrt{u^{2}+v^{2}} e^{-x \tau} \cos (-y \tau+\varphi)=0, \\
& y+\sqrt{u^{2}+v^{2}} e^{-x \tau} \sin (-y \tau+\varphi)=0,
\end{aligned}
$$

where $\varphi=\arg \left(\lambda_{k}\right)=\arctan (v / u)$ is the argument of $\lambda$ and $\varphi \in(-\pi / 2, \pi / 2)$.

According to (22), we can know that

$$
\begin{gathered}
x^{2}+y^{2}=\left(u^{2}+v^{2}\right) e^{-2 x \tau}, \\
y \tau= \pm \tau \sqrt{\left(u^{2}+v^{2}\right) e^{-2 x \tau}-x^{2}} .
\end{gathered}
$$

Substituting those into the first equation of (22), we have

$$
x+\sqrt{u^{2}+v^{2}} e^{-x \tau} \cos \left(\mp \tau \sqrt{\left(u^{2}+v^{2}\right) e^{-2 x \tau}-x^{2}}+\varphi\right)=0 ;
$$

that is,

$$
x+\sqrt{u^{2}+v^{2}} e^{-x \tau} \cos \left(\tau \sqrt{\left(u^{2}+v^{2}\right) e^{-2 x \tau}-x^{2}} \pm \varphi\right)=0 .
$$

Let $x$ be equal to the critical value; that is, $x=0$; then

$$
\tau \sqrt{\left(u^{2}+v^{2}\right)} \pm \varphi=\frac{\pi}{2}+m \pi, \quad m=0, \pm 1, \pm 2, \ldots
$$

In order to get the smallest positive value of $\tau$, we have $m=0$, and $\tau \sqrt{\left(u^{2}+v^{2}\right)}+|\varphi|=\pi / 2$, since $\tau \sqrt{\left(u^{2}+v^{2}\right)} \geq 0$ and the sign of $\varphi$ is decided by $u$ and $v$. So, we can get the critical value of the time delay, denoted as $\tau_{0}$, and

$$
\tau_{0}=\frac{\pi / 2-|\varphi|}{\sqrt{u^{2}+v^{2}}} .
$$

In the following, we will show that the roots of (17) have negative real part when $\tau<\tau_{0}$.

Since the complex eigenvalues of $L$ are conjugated, their imaginary signs arguments are opposite. According to (25), we define the function as

$$
\begin{aligned}
& f(x) \\
& \quad=x+\sqrt{u^{2}+v^{2}} e^{-x \tau} \cos \left(\tau \sqrt{\left(u^{2}+v^{2}\right) e^{-2 x \tau}-x^{2}}+|\varphi|\right) .
\end{aligned}
$$

For arbitrary $\tau_{1}>(\pi / 2-|\varphi|) /\left(\sqrt{u^{2}+v^{2}}\right)$, let $x=0$, and if $\tau=\tau_{1}$, then

$$
f(0)=\sqrt{u^{2}+v^{2}} \cos \left(\tau_{1} \sqrt{u^{2}+v^{2}}+|\varphi|\right)<0 .
$$

For function $\sqrt{\left(u^{2}+v^{2}\right) e^{-2 x \tau_{1}}-x^{2}}$, there exists a positive root $b>0$, and it decreases monotonically in the interval $[0, b]$.

Taking

$$
\tau_{1} \sqrt{\left(u^{2}+v^{2}\right) e^{-2 x \tau_{1}}-x^{2}}+|\varphi|=\frac{\pi}{2}
$$

there exists only one solution in the interval $(0, b)$, denoted as $x_{1}$.

Then

$$
f\left(x_{1}\right)=x_{1}>0 \text {. }
$$

From (29) and (31), there exists a root of $f(x)$ between 0 and $x_{1}$. Moreover, if $\tau=\tau_{1}$, the same result holds.

For arbitrary $\tau_{2}<\tau_{0}$, then

$$
f(0)=\sqrt{u^{2}+v^{2}} \cos \left(\tau_{2} \sqrt{u^{2}+v^{2}}+|\varphi|\right)>0 .
$$


Because $\sqrt{\left(u^{2}+v^{2}\right) e^{-2 x \tau_{2}}-x^{2}}$ is a monotonically decreasing function in the interval $(0, b), 0<\tau_{2} \sqrt{\left(u^{2}+v^{2}\right) e^{-2 x \tau_{2}}-x^{2}}+$ $|\varphi|<\pi / 2$ and $f(x)>0,(x \in(0, b))$, which implies that they have no positive roots of (28) but have infinite negative roots of (28).

Define

$$
h=\min _{k=\{2,3, \ldots, n\}} \frac{\pi / 2-\left|\arg \left(\lambda_{k}\right)\right|}{\left\|\lambda_{k}\right\|},
$$

for arbitrary $\tau<h$; the roots of the denominator of each term of (15) must have negative real part.

Take arbitrary term of (15), simply denoted as

$$
G(s)=\frac{c}{\left(s+\lambda e^{-s \tau}\right)^{r}},
$$

where $c$ is the coefficient, $r$ is the degree, and $\tau<h$. It has infinite singular points which have negative real part. The Laplace inverse transform of the function asymptotically tends to 0 ; that is, for the function,

$$
g(t)=\mathscr{L}^{-1}(G(s)),
$$

when $t \rightarrow+\infty$ and $g(t) \rightarrow 0$. So, we can obtain

$$
x_{i}(t)=\mathscr{L}^{-1}\left(\mathscr{X}_{i}(s)\right) \longrightarrow c_{1}^{i},
$$

when $t \rightarrow+\infty$; that is, $X(t) \rightarrow\left(c_{1}^{1}, c_{2}^{1}, \ldots, c_{n}^{1}\right)^{T}$ as $t \rightarrow+\infty$.

According to Lemma 1, we can get

$$
\begin{aligned}
& c=c_{1}^{1}=c_{1}^{2}=\cdots=c_{1}^{n}
\end{aligned}
$$

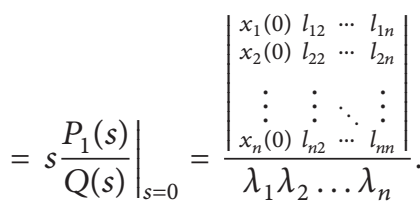

This completes the proof.

Remark 5. If matrix $A$ is symmetrical, then the imaginary parts of the eigenvalues are equal to zero; that is, $\varphi_{k}=0$. The critical value of the time delay is $h=\pi / 2 \lambda_{\max }$.

Furthermore, the multiagent system is described by

$$
\begin{array}{r}
\dot{x}_{i}(t)=-\sum_{j \in \mathcal{N}_{i}} a_{i j}\left(x_{i}\left(t-\tau_{j i}(t)\right)-x_{j}\left(t-\tau_{i j}(t)\right)\right) \\
i=1,2, \ldots, n .
\end{array}
$$

We can also have the same result as follows.

Theorem 6. For model (38), if there exists at least one globally reachable node in digraph $\mathscr{G}$ and the time delay parameter $\tau$ satisfies

$$
\tau(t)<\min _{i=2,3, \ldots, n} \frac{\pi / 2-\left|\arg \left(\lambda_{i}\right)\right|}{\left\|\lambda_{i}\right\|},
$$

then all the agents of the system can achieve asymptotically consensus.
In model (5), we regarded the feedback gain value as 1 . If the feedback gain value is $a$, then the model can be described as

$$
\dot{x}_{i}(t)=-a \sum_{j \in \mathcal{N}_{i}} a_{i j}\left(x_{i}(t-\tau)-x_{j}(t-\tau)\right), \quad i=1,2, \ldots, n,
$$

which can be rewritten as the matrix form

$$
\dot{X}(t)=-a L X(t-\tau) .
$$

It can be easy to get that the consensus center is not changed; then, we have the following theorem.

Theorem 7. For model (40), if there exists at least one reserve tree in the digraph $\mathscr{G}$ associated with matrix $A$ and the time delay parameter $\tau$ satisfies

$$
\tau<\min _{i=2,3, \ldots, n} \frac{\pi / 2-\left|\arg \left(\lambda_{i}\right)\right|}{a\left\|\lambda_{i}\right\|},
$$

then all the agents of the system will asymptotically converge upon a fixed point $c$, where

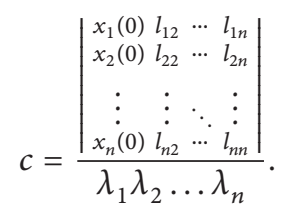

Remark 8. The feedback gain $a$ can be used to adjust the consensus velocity of the agents and the critical value of delay.

\section{Numerical Simulations}

In this section, we will give some numerical simulations to illustrate the theoretical results.

Consider a multiagent system with ten agents, where the initial states of agents are chosen randomly. The coupling matrices are given as follows, respectively:

$$
A_{1}=\left(\begin{array}{llllllllll}
0 & 0 & 0 & 0 & 0 & 0 & 0 & 0 & 0 & 0 \\
1 & 0 & 0 & 0 & 0 & 0 & 0 & 0 & 0 & 0 \\
1 & 0 & 0 & 0 & 0 & 0 & 0 & 0 & 0 & 0 \\
1 & 0 & 0 & 0 & 0 & 0 & 0 & 0 & 0 & 0 \\
1 & 0 & 0 & 0 & 0 & 0 & 0 & 0 & 0 & 0 \\
1 & 0 & 0 & 0 & 0 & 0 & 0 & 0 & 0 & 0 \\
1 & 0 & 0 & 0 & 0 & 0 & 0 & 0 & 0 & 0 \\
1 & 0 & 0 & 0 & 0 & 0 & 0 & 0 & 0 & 0 \\
1 & 0 & 0 & 0 & 0 & 0 & 0 & 0 & 0 & 0 \\
1 & 0 & 0 & 0 & 0 & 0 & 0 & 0 & 0 & 0
\end{array}\right),
$$

$$
A_{2}=\left(\begin{array}{llllllllll}
0 & 0 & 0 & 0 & 0 & 0 & 0 & 0 & 0 & 1 \\
1 & 0 & 0 & 0 & 0 & 0 & 0 & 0 & 0 & 0 \\
0 & 1 & 0 & 0 & 0 & 0 & 0 & 0 & 0 & 0 \\
0 & 0 & 1 & 0 & 0 & 0 & 0 & 0 & 0 & 0 \\
0 & 0 & 0 & 1 & 0 & 0 & 0 & 0 & 0 & 0 \\
0 & 0 & 0 & 0 & 1 & 0 & 0 & 0 & 0 & 0 \\
0 & 0 & 0 & 0 & 0 & 1 & 0 & 0 & 0 & 0 \\
0 & 0 & 0 & 0 & 0 & 0 & 1 & 0 & 0 & 0 \\
0 & 0 & 0 & 0 & 0 & 0 & 0 & 1 & 0 & 0 \\
0 & 0 & 0 & 0 & 0 & 0 & 0 & 0 & 1 & 0
\end{array}\right)
$$



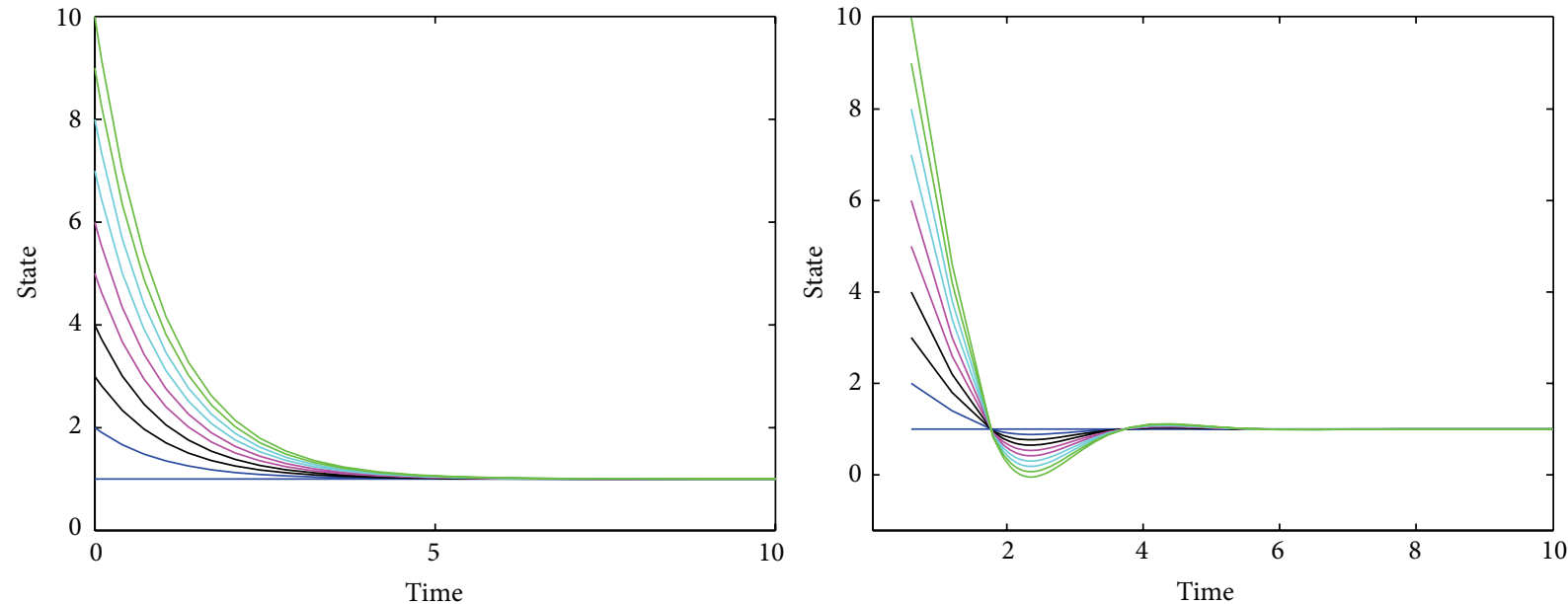

FIGURE 1: States of the agents about model (5) with the coupling matrix $A_{1}$, when $\tau=0$ and 0.6 , respectively.
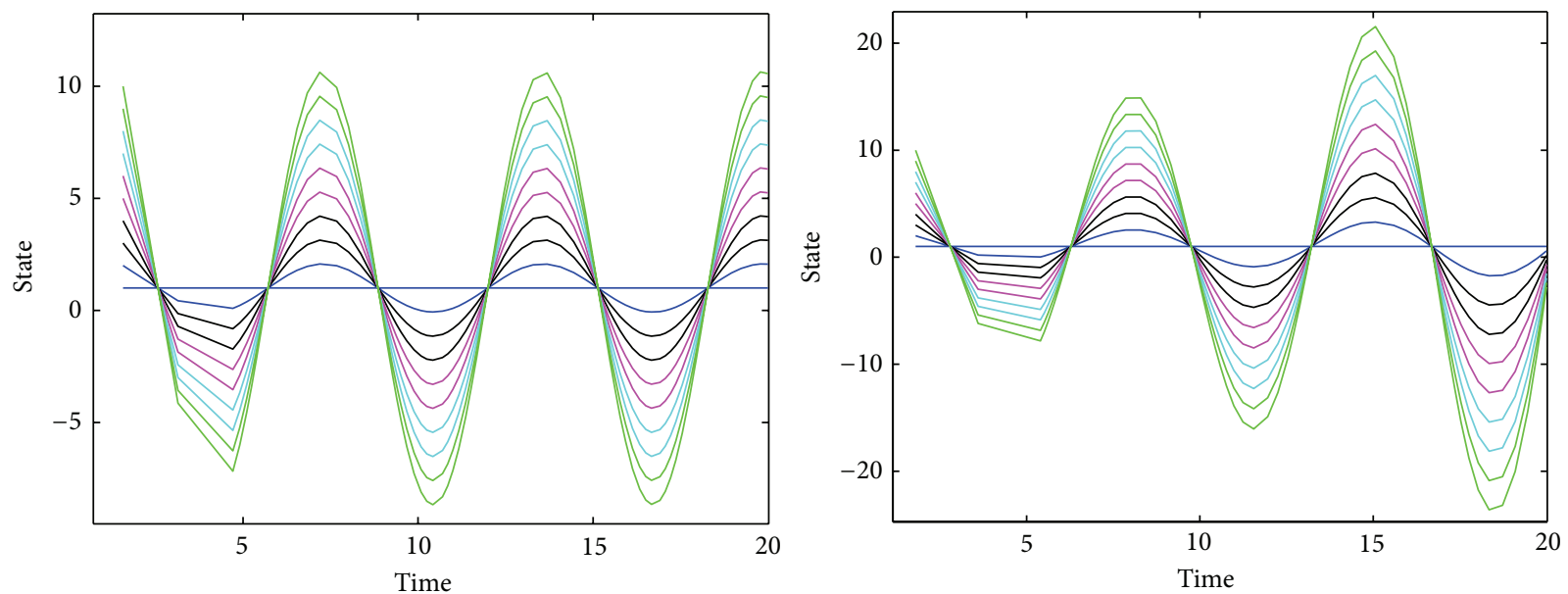

FIGURE 2: States of the agents about model (5) with the coupling matrix $A_{1}$, when $\tau=\pi / 2$ and 1.8 , respectively.
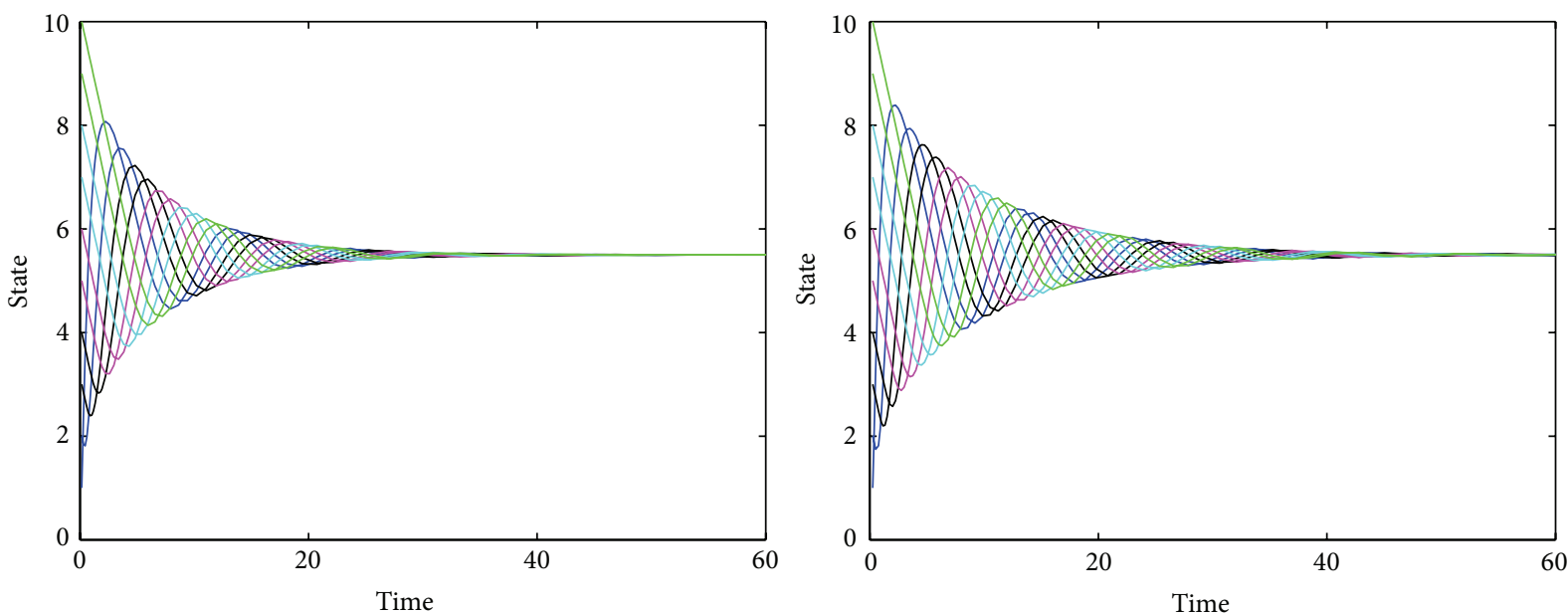

Figure 3: States of the agents about model (38) with the coupling matrix $A_{2}$ and the feedback gain $a=0.5$, when $\tau=0.3$ and 0.5089 , respectively. 
Matrix $A_{1}$ is the associated Laplacian matrix with only one zero eigenvalue, and the other eigenvalues are all equal to 1 . And the critical value of delay is $h_{1}=\pi / 2$.

For matrix $A_{2}$, the associated digraph $\mathscr{G}$ is strongly connected, and the eigenvalues of $L$ are

$$
\begin{aligned}
& 0.0000,2.0000,1.8090+0.5878 i, 1.8090-0.5878 i ; \\
& 1.3090+0.9511 i, 1.3090-0.9511 i, 0.6910+0.9511 i \\
& 0.6910-0.9511 i, 0.1910+0.5878 i, 0.1910-0.5878 i
\end{aligned}
$$

The critical value of delay $h_{3}=\min _{i=2,3, \ldots, n}((\pi / 2-$ $\left.\left.\left|\arg \left(\lambda_{i}\right)\right|\right) / a\left\|\lambda_{i}\right\|\right)=0.5089$.

Figures 1 and 2 show the simulation results, where the coupling matrix is $A_{1}$. We can find that, from Figure 1 , the states of all agents of model (5) will asymptotically converge to a constant value when $\tau=0,0.6(\tau<\pi / 2)$, respectively; however, from Figure 2, the states of all agents of the system will diverge when $\tau=\pi / 2,1.8(\tau \geq \pi / 2)$, respectively. Figure 3 shows, for model (38), the states of all agents of the system with the coupling matrix $A_{2}$, and the feedback gain will asymptotically converge to a constant value when $\tau=0.3,0.5089(\tau<\pi / 2)$, respectively.

\section{Conclusion}

In this paper, we have considered the consensus of multiagent systems with directed topology and communication time delay. We have proved that the system aggregates and forms a cluster in finite time if the time delays are smaller than the critical value. The methods and results of this paper can be extended to discuss the leader-follower second-order multiagent system with time delay.

\section{Conflict of Interests}

The authors declare that there is no conflict of interests regarding the publication of this paper.

\section{Acknowledgments}

This work was supported by the National Natural Science Foundation of China under Grant (nos. 61304049, 61174116, and 51308005) and Science and Technology Development Plan Project of Beijing Education Commission (no. KM201310009011).

\section{References}

[1] T. Vicsek, A. Czirk, E. Ben-Jacob, I. Cohen, and O. Shochet, "Novel type of phase transition in a system of self-driven particles," Physical Review Letters, vol. 75, no. 6, pp. 1226-1229, 1995.

[2] H. Su, X. Wang, and G. Chen, "Rendezvous of multiple mobile agents with preserved network connectivity," Systems \& Control Letters, vol. 59, no. 5, pp. 313-322, 2010.

[3] A. Jadbabaie, J. Lin, and A. S. Morse, "Coordination of groups of mobile autonomous agents using nearest neighbor rules," IEEE Transactions on Automatic Control, vol. 48, no. 6, pp. 988-1001, 2003.
[4] H. Su, G. Chen, X. Wang, and Z. Lin, "Adaptive secondorder consensus of networked mobile agents with nonlinear dynamics," Automatica, vol. 47, no. 2, pp. 368-375, 2011.

[5] R. Olfati-Saber and R. M. Murray, "Consensus problems in networks of agents with switching topology and time-delays," IEEE Transactions on Automatic Control, vol. 49, no. 9, pp. 15201533, 2004.

[6] H. Su, X. Wang, and G. Chen, "A connectivity-preserving flocking algorithm for multi-agent systems based only on position measurements," International Journal of Control, vol. 82, no. 7, pp. 1334-1343, 2009.

[7] J. Hu and Y. Hong, "Leader-following coordination of multiagent systems with coupling time delays," Physica A: Statistical Mechanics and its Applications, vol. 374, no. 2, pp. 853-863, 2007.

[8] H. Su, X. Wang, and Z. Lin, "Flocking of multi-agents with a virtual leader," IEEE Transactions on Automatic Control, vol. 54, no. 2, pp. 293-307, 2009.

[9] J. Wang, J. Hu, Y. Hong, and D. Cheng, "Consensus of a class of multi-agent systems with active leader and time delay," Graduate School of Chinese Academy of Sciences, vol. 25, no. 3, pp. 320-328, 2008.

[10] H. Su, N. Zhang, M. Z. Q. Chen, and X. Wang, "Adaptive flocking with a virtual leader of multiple agents governed by locally Lipschitz nonlinearity," Nonlinear Analysis: Real World Applications, vol. 14, no. 1, pp. 798-806, 2013.

[11] W. Ren and R. W. Beard, "Consensus seeking in multiagent systems under dynamically changing interaction topologies," IEEE Transactions on Automatic Control, vol. 50, no. 5, pp. 655661, 2005.

[12] Z. Lin, B. Francis, and M. Maggiore, "Necessary and sufficient graphical conditions for formation control of unicycles," IEEE Transactions on Automatic Control, vol. 50, no. 1, pp. 121-127, 2005.

[13] H. Su, M. Chen, X. Wang, and J. Lam, "Semiglobal observerbased leader-following consensus with input saturation," IEEE Transactions on Industrial Electronics, vol. 61, no. 6, pp. 28422850, 2014.

[14] H. Su, Z. Rong, M. Z. Q. Chen, X. Wang, G. Chen, and H. Wang, "Decentralized adaptive pinning control for cluster synchronization of complex dynamical networks," IEEE Transactions on Systems, Man, and Cybernetics B: Cybernetics, vol. 43, no. 1, pp. 394-399, 2013.

[15] H. Su, M. Z. Q. Chen, J. Lam, and Z. Lin, "Semi-global leaderfollowing consensus of linear multi-agent systems with input saturation via low gain feedback," IEEE Transactions on Circuits and Systems I, vol. 60, no. 7, pp. 1881-1889, 2013.

[16] L. Ji and X. Liao, "Consensus problems of first-order dynamic multi-agent systems with multiple time delays," Chinese Physics B, vol. 22, no. 4, Article ID 040203, 2013. 


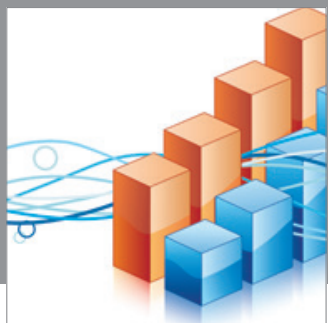

Advances in

Operations Research

mansans

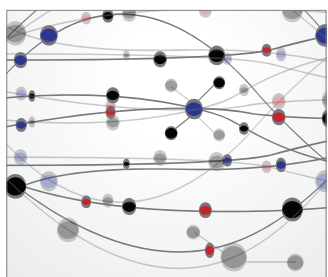

The Scientific World Journal
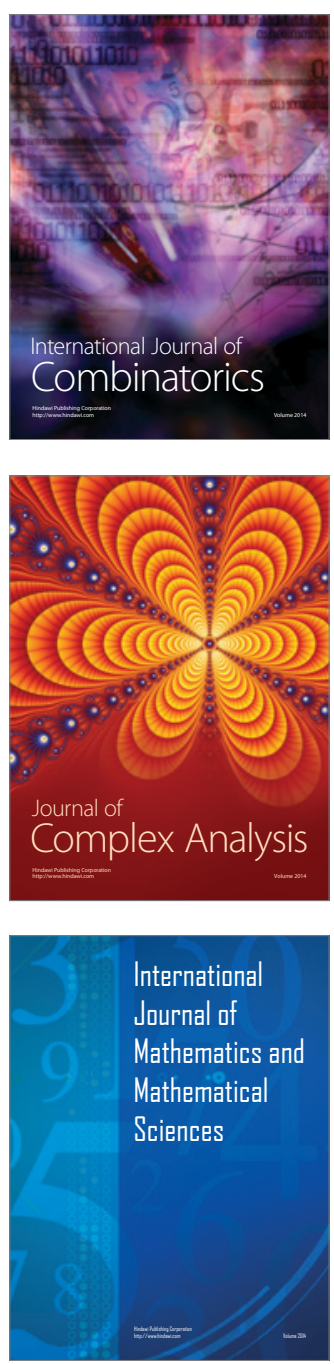
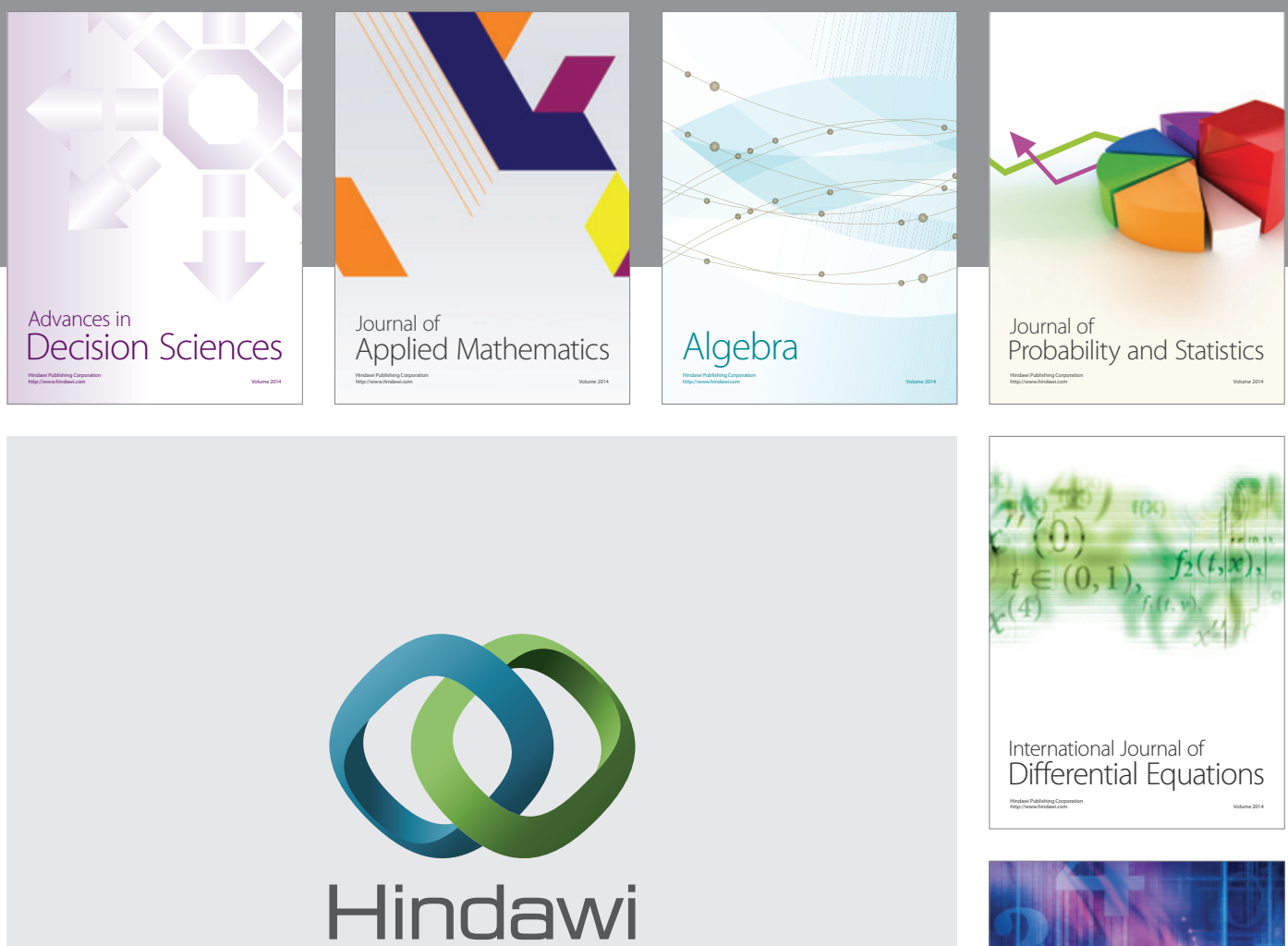

Submit your manuscripts at http://www.hindawi.com
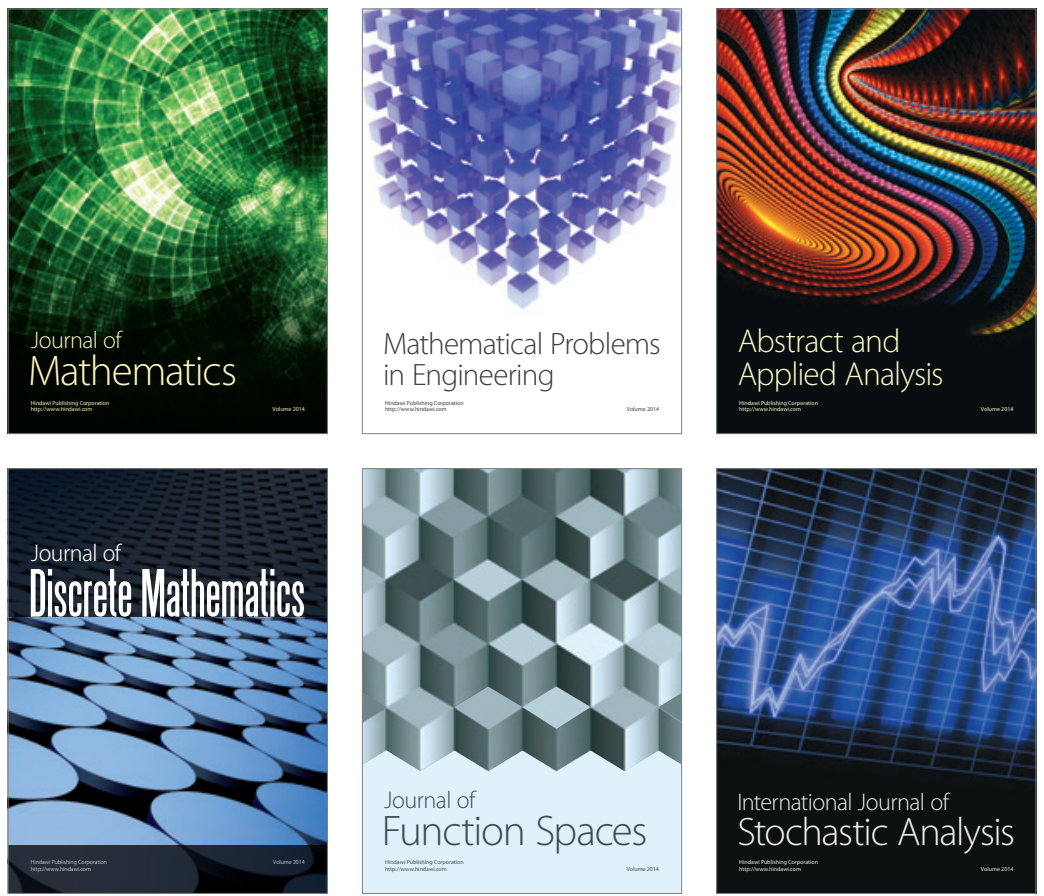

Journal of

Function Spaces

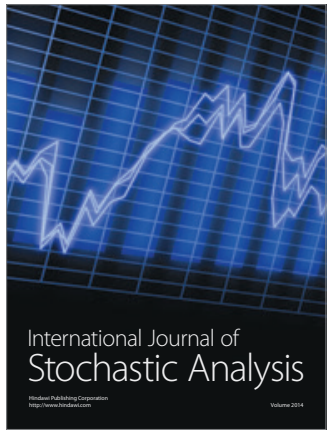

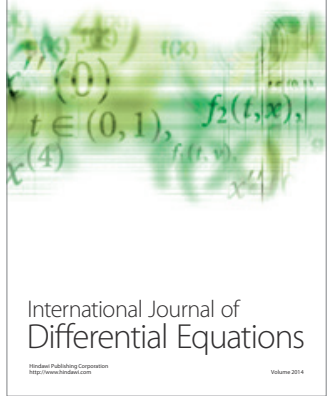
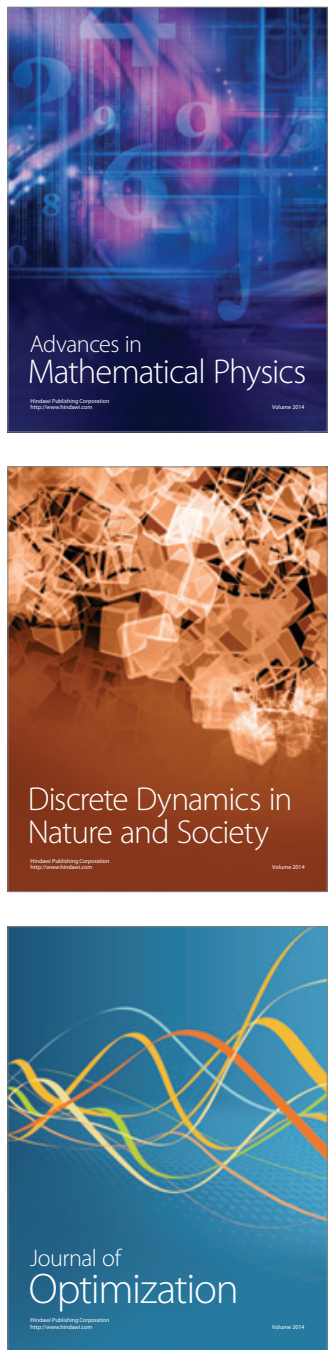